\title{
Localized Radiative Energy Transfer from a Plasmonic Bow-Tie Nanoantenna to a Magnetic Thin Film Stack
}

\author{
K. Sendur ${ }^{1}$, A. Kosar ${ }^{1}$, and M. P. Menguc ${ }^{2,3}$ \\ ${ }^{1}$ Sabanci University, Orhanli-Tuzla, 34956, Istanbul, Turkey \\ ${ }^{2}$ Ozyegin University, Uskudar, 34662 Istanbul, Turkey \\ ${ }^{3}$ University of Kentucky, Lexington, KY 40506, USA \\ sendur@sabanciuniv.edu
}

\begin{abstract}
-
Localized radiative energy transfer from a near-field emitter to a magnetic thin film structure is investigated. A magnetic thin film stack is placed in the near-field of the plasmonic nanoantenna to utilize the evanescent mode coupling between the nanoantenna and magnetic thin film stack. A bow-tie nano-optical antenna is excited with a tightly focused beam of light to improve near-field radiative energy transfer from the antenna to the magnetic thin film structure. A tightly focused incident optical beam with a wide angular spectrum is formulated using Richards-Wolf vector field equations. Radiative energy transfer is investigated using a frequency domain 3-D finite element method solution of Maxwell's equations. Localized radiative energy transfer between the near-field emitter and the magnetic thin film structure is quantified for a given optical laser power at various distances between the near-field emitter and magnetic thin film.
\end{abstract}

\section{INTRODUCTION}

Emerging nano-scale plasmonic applications, such as heat-assisted magnetic recording (HAMR), require localized radiative energy transfer that can be achieved using intense optical spots beyond the diffraction limit $[1,2,3]$. When objects are separated by less than a subwavelength scale, the radiative energy transfer between the surfaces can be several orders higher than predicted by Planck's blackbody radiation. The drastic improvement of the radiative energy transfer has potential applications in emerging technologies including heat-assisted magnetic recording [1, 2, 3], thermophotovoltaic energy devices [4, 5], and optically-assisted nanomanufacturing [6, 7].

Recently, there has been increasing research on the radiative energy exchange between object separated by less than a subwavelength scale. In recent studies, Francoeur et al. [8, 9] and Shen et al. [10] demonstrated that the surface phonon polaritons improve the radiative energy transfer between two surfaces at small gaps. Rousseau et al. [11] investigated an experimental setup that can measure the conductance when the objects are separated by varying the distances between 30 nm and $2.5 \mu \mathrm{m}$. The experimental data by Rousseau et al. [11] confirms the theoretical results that the near-field radiative energy transfer can be substantially improved at the nanoscale. These studies have demonstrated the potential for engineering near-field emitters for potential applications. Additionally, it has been shown that the radiative energy transfer can be tuned via multiple thin films supporting surface plasmon polaritons $[12,13,14,15]$. Laser-induced radiative energy transfer involving a scanning probe microscope tip has also been investigated in the literature [16, 17].

An optical nano-antenna can be utilized as a near-field emitter for radiative energy transfer to an object placed in the close vicinity. Antennas have been an efficient means to couple incident electromagnetic energy with small scale electronic devices. A similar coupling mechanism is applicable at optical frequencies between nano-antennas operating at optical frequencies and objects with feature dimensions below the diffraction limit [18, 19, 20, 21, 22, 23]. At optical frequencies, a nanoscale metallic antenna can be utilized to couple an incident optical beam to length scales much smaller than the diffraction limit.

In this study, the radiative energy transfer from a gold bow-tie nano-antenna to a magnetic thin film layer is investigated. A tightly focused beam of light with a wide angular spectrum illuminates the near-field emitter. In Section 2, the formulation for the focused beam model based on RichardsWolf vector field theory and details of the numerical calculations are presented. In Section 3, the radiative energy transfer between the near-field emitter and the magnetic thin film structure is studied. Localized radiative energy transfer between the emitter and the thin film structure is quantified for a given optical laser power at various distances between them. Concluding remarks appear in Section 4. 


\section{THEORY}

For enhanced localized radiative energy transfer to a magnetic thin-film stack, a bow-tie antenna is used in this study. A bow-tie antenna in the vicinity of the magnetic thin-film structure is illustrated in Fig. 1. An oblique view of the problem geometry is illustrated in Fig. 1(a). Details of the magnetic thin-film stack and some physical parameters regarding the antenna geometry that will be investigated in this study are shown in Fig. 1(b). The bow-tie antenna consists of two triangular gold particles that are separated with a distance of $G$ as shown in Fig. 1. The physical separation between the antenna and the magnetic thin film is $D$.

(a)

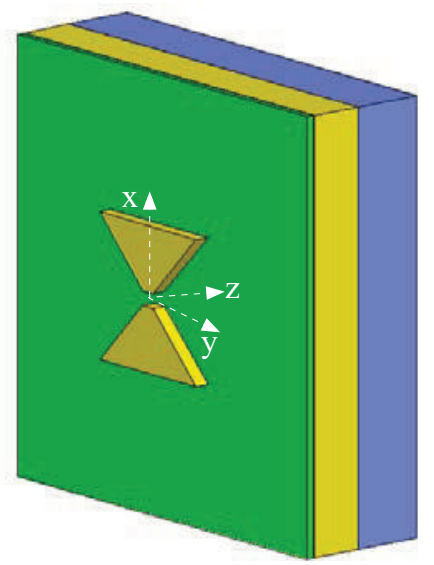

(b)

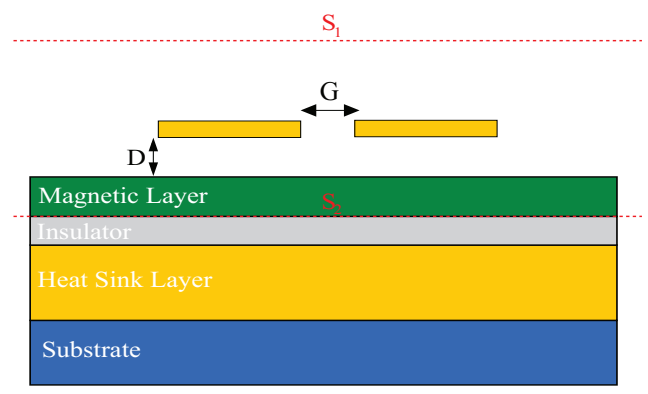

Figure 1: (a) An oblique view of the problem geometry involving a bow-tie antenna and a magnetic thin-film stack. (b) A cross section shows the details of the magnetic thin-film and the antenna's geometry.

The nano-antenna in this study is excited using a focused beam of light from an optical lens. The propagating direction of the incident beam is in the $\hat{z}$-direction. To model the incident focused beam, we used the Richards-Wolf theory $[24,25]$. A tightly focused beam of light has a wide angular spectrum. The Richards-Wolf theory $[24,25]$ provides an accurate representation of an incident beam near the focus of an aplanatic lens. Using the Richards-Wolf vector field representation $[24,25]$ the total electric field in the vicinity of the focus is given as

$$
\mathbf{E}\left(\mathbf{r}_{p}\right)=-\frac{i}{\lambda} \int_{0}^{\alpha} d \theta \sin \theta \int_{0}^{2 \pi} d \phi \mathbf{a}(\theta, \phi) \exp \left(-i \mathbf{k} \cdot \mathbf{r}_{p}\right)
$$

where $\alpha$ is the half angle of the beam, $\mathbf{r}_{p}$ is the observation point

$$
\mathbf{r}_{p}=x_{p} \hat{x}+y_{p} \hat{y}+z_{p} \hat{z}=r_{p} \cos \phi_{p} \hat{x}+r_{p} \sin \phi_{p} \hat{y}+z_{p} \hat{z}
$$

and

$$
\mathbf{k}=\frac{2 \pi}{\lambda}(\sin \theta \cos \phi \hat{x}+\sin \theta \sin \phi \hat{y}-\cos \theta \hat{z}) .
$$

In Eqs. (2) and (3) $\lambda$ is the wavelength, $r_{p}=\sqrt{x_{p}^{2}+y_{p}^{2}}$, and $\phi_{p}=\arctan \left(y_{p} / x_{p}\right)$. In Eq. (1), a $(\theta, \phi)$ is the weighting vector, which is given as

$$
\mathbf{a}(\theta, \phi)=\left[\begin{array}{c}
\cos \theta \cos ^{2} \phi+\sin ^{2} \phi \\
\cos \theta \cos \phi \sin \phi-\cos \phi \sin \phi \\
\sin \theta \cos \phi
\end{array}\right] \sqrt{\cos \theta},
$$

The power of the incident beam propagating in the $\hat{z}$ direction is given by the Poynting's vector

$$
P_{z}=\operatorname{Re}\left[\iint_{S_{1}}\left(\frac{1}{2} \mathbf{E}^{\mathbf{i}}(\mathbf{r}) \times \mathbf{H}^{\mathbf{i} *}(\mathbf{r})\right) \cdot \hat{n}_{1} d S\right]
$$

where $\mathbf{E}^{\mathbf{i}}(\mathbf{r})$ is the incident electric field given by Eq. (1) and the $\mathbf{H}^{\mathbf{i}}(\mathbf{r})$ is obtained using Maxwell's equation. The integration surface $S_{1}$, shown in Fig. 1(b), is perpendicular to the direction of propagation. 
The incident electric field, given by Eq. (1), represents the optical beam generated by the lens system in the absence of the nano-antenna and magnetic thin film stack. Once the incident electric field interacts with the antenna and the magnetic thin film stack, scattered fields $\mathbf{E}^{s}(\mathbf{r})$ are generated. The total electric field $\mathbf{E}^{t}(\mathbf{r})$ is composed of two components

$$
\mathbf{E}^{t}(\mathbf{r})=\mathbf{E}^{i}(\mathbf{r})+\mathbf{E}^{s}(\mathbf{r})
$$

where $\mathbf{E}^{t}(\mathbf{r}), \mathbf{E}^{i}(\mathbf{r})$, and $\mathbf{E}^{s}(\mathbf{r})$ are the total, incident, and scattered electric field components, respectively. In this study, to obtain the scattered field, $\mathbf{E}^{s}(\mathbf{r})$, we used a 3-D finite element method (FEM) based full-wave solution of Maxwells equations. To represent the scattering geometries accurately, tetrahedral elements are used to discretize the computational domain. Radiation boundary conditions are used in FEM simulations. On the tetrahedral elements, edge basis functions and second-order interpolation functions are used to expand the functions. Adaptive mesh refinement is used to improve the coarse solution regions with high field intensities and large field gradients. Adaptive mesh refinement helps to obtain accurate results for different types of geometries. Once the scattered field is solved via the FEM, the total field can be obtained using Eq. (6).

Conversion of the field quantities into power quantities is achieved by applying the Poyntings theorem $[27,28]$ to the geometry given in Fig 1 . The dissipated power within the sample due to the near-field electromagnetic radiation can be obtained by utilizing Poynting's theorem $[27,28]$

$$
\begin{aligned}
\iiint_{V_{M}} \sigma|\mathbf{E}(\mathbf{r})|^{2} d V & =\iint_{S_{1}}\left(\frac{1}{2} \mathbf{E}(\mathbf{r}) \times \mathbf{H}^{*}(\mathbf{r})\right) \cdot \hat{n}_{1} d S-\iint_{S_{2}}\left(\frac{1}{2} \mathbf{E}(\mathbf{r}) \times \mathbf{H}^{*}(\mathbf{r})\right) \cdot \hat{n}_{2} d S \\
& -\iiint_{V_{A}} \sigma|\mathbf{E}(\mathbf{r})|^{2} d V-2 i w \iiint_{V}\left(\frac{1}{4} \epsilon|\mathbf{E}(\mathbf{r})|^{2}+\frac{1}{4} \mu|\mathbf{H}(\mathbf{r})|^{2}\right) d V
\end{aligned}
$$

where $V_{M}$ represents the magnetic layer, $V_{A}$ represents the antenna particles, and $S_{1}$ and $S_{2}$ are the surfaces perpendicular to the direction of propagation shown in Fig. 1. The term on the lefthand side of the equation represents the optical power dissipated over the magnetic film. The first and second terms on the right hand side of the equation represents the optical power entering and exiting the structures from surfaces $S_{1}$ and $S_{2}$, respectively. The third term represents the optical power dissipated over antenna particles and the last term represents the complex reactive energy stored in the volume between $S_{1}$ and $S_{2}$. Eq. (7) along with Eq. (1) and Eq. (6) will be utilized to obtain the optical dissipated power profiles over the magnetic film.

\section{RESULTS}

In this study, the power of the incident optical beam on the antenna is taken as $P_{z}=100 \mathrm{~mW}$ and the operating wavelength is $700 \mathrm{~nm}$. The half-beam angle $\alpha$ is selected as $60^{\circ}$, which corresponds to a numerical aperture of about 0.86 . The intensity distribution of the incident optical beam onto the nanoantenna is illustrated in Fig. 2(a) for an input power of $100 \mathrm{~mW}$. The optical beam shown in Fig. 2(a) is linearly polarized in the $\hat{x}$ direction. The direction of the polarization of the incident radiation and the orientation of the antenna geometry play an important role in the radiative energy transfer process. If the incident polarization is along the long-axis of the antenna as shown in Fig. 1, then the incident electromagnetic radiation creates induced currents along this axis in the antenna. These induced currents are the source of charge accumulation at the ends of the antenna. The charges created across the gap separating the metallic parts of the antenna have opposite polarity. The oscillation of the charges with opposite polarity is the source of localized radiative energy transfer to the magnetic thin film stack.

The thickness of the gold antenna is selected as $20 \mathrm{~nm}$ and the antenna particles are separated by $20 \mathrm{~nm}$. The magnetic thin film stack is composed of a $10 \mathrm{~nm}$ magnetic layer, $2 \mathrm{~nm}$ insulator layer, $100 \mathrm{~nm}$ heat-sink layer deposited over a substrate. The dielectric constants of metals at different wavelength are obtained from Palik [26]. The dissipated optical power density profile in the magnetic recording medium is given in Fig. 2(b) when the antenna is placed $10 \mathrm{~nm}$ away from the magnetic thin film stack. The results indicate that a strong localized radiative energy transfer is obtained between the antenna and magnetic thin film.

An important parameter in the radiative energy transfer from the antenna to the magnetic thin film stack is the distance between the antenna and magnetic thin film. In Fig. 3, localized radiative 


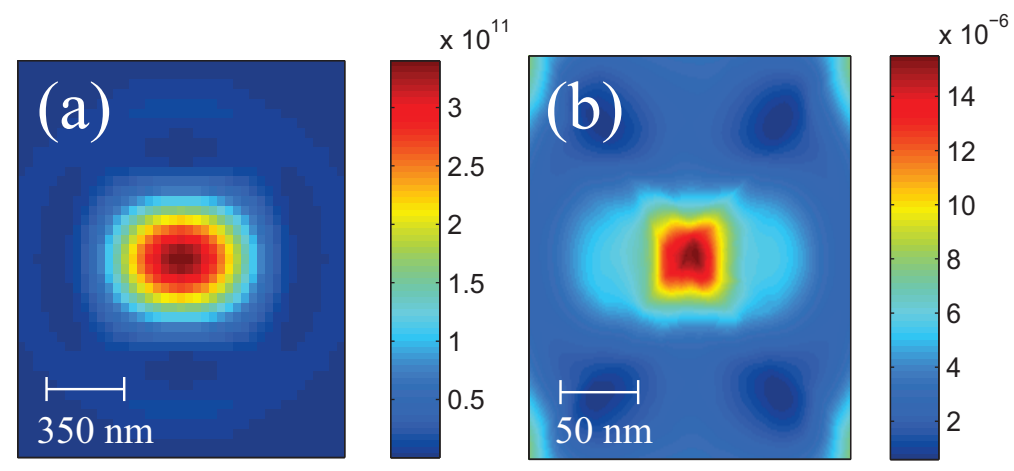

Figure 2: (a) Intensity distribution $\left[\mathrm{V}^{2} / \mathrm{m}^{2}\right]$ of the incident optical beam onto the nanoantenna. (b) Dissipated optical power density profile $\left[\mathrm{mW} / \mathrm{nm}^{3}\right]$ in the cobalt magnetic recording medium.

energy transfer between the near-field emitter and the magnetic thin film structure is quantified at various distances between the near-field emitter and magnetic thin film. The results show the sharp drop in the radiative energy transfer from the antenna to the magnetic thin film. The gold bow-tie nano-antenna primarily utilizes evanescent waves to couple optical energy to the magnetic thin film stack. As the distance is increased from $4 \mathrm{~nm}$ to $15 \mathrm{~nm}$, the dissipated power density reduces from $2.4 \times 10^{-5} \mathrm{~mW} / \mathrm{nm}^{3}$ to $1.3 \times 10^{-5} \mathrm{~mW} / \mathrm{nm}^{3}$. It is obvious that the evanescent component of the electromagnetic field is particularly important in large radiative energy transfer from the nanoantenna.

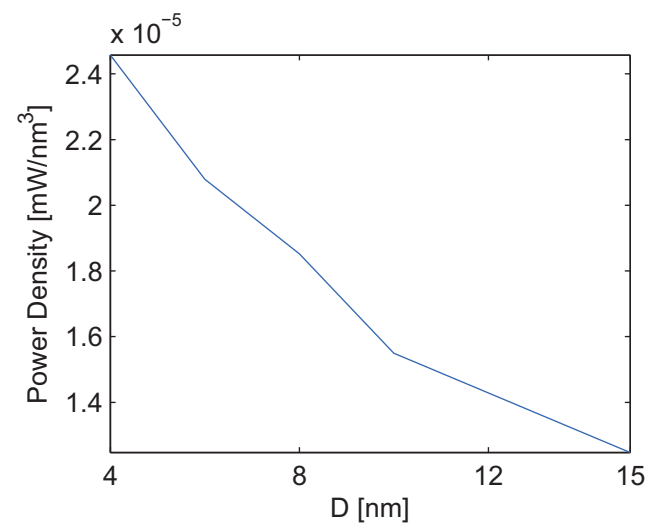

Figure 3: Dissipated optical power density $\left[\mathrm{mW} / \mathrm{nm}^{3}\right]$ in the cobalt magnetic recording medium as a function of distance from the nanoantenna.

\section{CONCLUSION}

Radiative energy transfer from a nanoscale bow-tie optical antenna to a magnetic thin film stack was investigated. The dissipated optical power density profile in the magnetic thin film stack shows a strong localized radiative energy transfer from the nanoantenna. Localized radiative energy transfer between the near-field emitter and the magnetic thin film structure was quantified for a given optical laser power at various distances between the near-field emitter and magnetic thin film.

\section{ACKNOWLEDGMENT}

This work is supported by TUBITAK under project number $108 \mathrm{~T} 482$ and by Marie Curie International Reintegration Grant (IRG) to Kursat Sendur (MIRG-CT-2007-203690) and to M. Pinar Menguc (NF-RAD (239382) through FP7-PEOPLE-IRG-2008). Kursat Sendur acknowledges partial support from the Turkish Academy of Sciences.

\section{REFERENCES}

1. Challener, W. A., C. Peng, A. V. Itagi, D. Karns, W. Peng, Y. Peng, X. Yang, X. Zhu, N. J. Gokemeijer, Y.-T. Hsia, G. Ju, R. E. Rottmayer, M. A. Seigler, and E. C. Gage, "Heat-assisted 
magnetic recording by a near-field transducer with efficient optical energy transfer," Nature Photon., Vol. 3, 220-224, 2009.

2. McDaniel, T., W. Challener, and K. Sendur, "Issues in heat assisted perpendicular recording," IEEE Trans. Mag., Vol. 39, 1972-1979, 2003.

3. Sendur, K., C. Peng, and W. Challener, "Near-field radiation from a ridge waveguide transducer in the vicinity of a solid immersion lens," Phys. Rev. Lett., Vol. 94, 043901, 2005.

4. Narayanaswamy, A. and G. Chen, "Surface modes for near field thermophotovoltaics," Appl. Phys. Lett., Vol. 82, 3544-3546, 2003.

5. DiMatteo, R. S., P. Greiff, S. L. Finberg, K. Young-Waithe, H. K. H. Choy, M. M. Masaki, and C. G. Fonstad, "Enhanced photogeneration of carriers in a semiconductor via coupling across a nonisothermal nanoscale vacuum gap," Appl. Phys. Lett., Vol. 79, 1894, 2001.

6. Lu, Y.-F., B. Hu, Z.-H. Mai, W.-J. Wang, W.-K. Chim, and T.-C. Chong, "Laser-Scanning Probe Microscope Based Nanoprocessing of Electronics Materials," Jpn. J. Appl. Phys., Vol. 40, 4395-4398, 2001.

7. Chimmalgi, A., C. P. Grigoropoulos, and K. Komvopoulos, "Surface nanostructuring by nano/femtosecond laser-assisted scanning force microscopy," J. Appl. Phys., Vol. 97, 104319, 2005.

8. Francoeur, M. and M. P. Menguc, "Role of the fluctuational electrodynamics theory in nearfield radiative heat transfer," J. Quant. Spectrosc. Radiat. Transfer, Vol. 109, 280-293, 2008.

9. Francoeur, M., M. P. Menguc, and R. Vaillon, "Near-field radiative heat transfer enhancement ia surface phonon-polaritons coupling in thin films," Appl. Phys. Lett., Vol. 93, 043109, 2008.

10. Shen, S., A. Narayanaswamy, and G. Chen, "Surface phonon polaritons mediated energy transfer between nanoscale gaps," Nano Lett., Vol. 9, 2909-2913, 2009.

11. Rousseau, E., A. Siria, G. Jourdan, S. Volz, F. Comin, J. Chevrier, and J.-J. Greffet, "Radiative heat transfer at the nanoscale," Nature Photon., Vol. 3, 514-517, 2009.

12. Ben-Abdallah, P., K. Joulain, J. Drevillon, and G. Domingues, "Tailoring the local density of states of nonradiative field at the surface of nanolayered materials," Appl. Phys. Lett., Vol. 94, 153117, 2009.

13. Francoeur, M., M. P. Menguc, and R. Vaillon, "Solution of near-field thermal radiation in onedimensional layered media using dyadic Greens functions and the scattering matrix method," J. Quant. Spectrosc. Radiat. Transfer, Vol 110, 2002-2018, 2009.

14. Francoeur, M., M. P. Menguc, and R. Vaillon, "Near-field radiative heat exchanges between two thin films supporting surface phonon-polaritons," J. Appl. Phys. (in press) 2010.

15. Francoeur, M., M. P. Menguc, and R. Vaillon, "Local density of electromagnetic states within a nanometric gap formed between two thin films supporting surface phonon-polaritons," $J$. Phys. D Appl. Phys. (in press) 2010.

16. Hawes, E. A., J. T. Hastings, C. Crofcheck, and M. P. Menguc, "Spatial Selective Melting and Evaporation of Nanosized Gold Particles," Opt. Lett., Vol. 33, 1383-1385, 2008.

17. Downes, A., D. Salter, and A. Elfick, "Heating effects in tip-enhanced optical microscopy," Opt. Express, Vol. 14, 5216-5222, 2006.

18. Grober, R. D., R. J. Schoelkopf, and D. E. Prober, "Optical antenna: Towards a unit efficiency near-field optical probe," Appl. Phys. Lett., Vol. 70, 1354-1356, 1997.

19. Sendur, K. and W. Challener, "Near-field radiation of bow-tie antennas and apertures at optical frequencies," J. Microsc., Vol. 210, 279-283, 2003.

20. Novotny, L., "Effective Wavelength Scaling for Optical Antennas," Phys. Rev. Lett., Vol. 98, 266802, 2007.

21. Crozier, K. B., A. Sundaramurthy, G. S. Kino, and C. F. Quate, "Optical antennas: Resonators for local field enhancement," J. Appl. Phys., Vol. 94, No. , 4632-4642, 2003.

22. Muhlschlegel, P., H.-J. Eisler, O. J. F. Martin, B. Hecht, and D. W. Pohl, "Resonant Optical Antennas," Science, Vol. 308, 1607-1609, 2005.

23. Sendur, K. and E. Baran, "Near-field optical power transmission of dipole nano-antennas," Appl. Phys. B, Vol. 96, 325-335, 2009.

24. Wolf, E., "Electromagnetic diffraction in optical systems I. An integral representation of the image field," Proc. Roy. Soc. London Ser. A, Vol. 253, 349-357, 1959.

25. Richards, B. and E. Wolf, "Electromagnetic diffraction in optical systems II. Structure of the image field in an aplanatic system," Proc. Roy. Soc. London Ser. A, Vol. 253, 358-379, 1959.

26. Palik, E. D., Handbook of optical constants of solids, Academic Press, San Diego, 1998.

27. Balanis, C. A., Advanced Engineering Electromagnetics, Wiley, New York, 1989.

28. Cheng, D. K., Field and Wave Electromagnetics, Addison-Wesley, New York, 1983. 